\title{
DOES PES PLANUS INFLUENCE STANDING BALANCE IN ELEMENTARY SCHOOL-AGE CHILDREN?
}

\author{
Mária Takács ${ }^{1}$, Gergely Nagymáté2, Rita M. Kiss² \\ ${ }^{1}$ Department of Orthopedics, MÁV Hospital Szolnok \\ ${ }^{2}$ Department of Mechatronics, Optics and Mechanical Engineering Informatics, \\ Budapest University of Technology and Economics
}

drtakacskmaria@freemail.hu

DOI: 10.17489/biohun/2019/1/01

\begin{abstract}
Purpose: No any research in literature was found to investigate the effect of pes planus on standing balance in school-aged children. Any kind of change in the arches (height, flexibility) may increase the possibility of a change in standing balance. The aim of present study is to determine the influence of pes planus on the standing balance of school-aged children based on independent time-distance and frequency based parameters.

Materials and Methods: Subjects included 177 children ( 105 neutral and 72 with pes planus). The parameters were determined from the motion of the centre of pressure (COP) on a platform equipped with pressure gauge sensors, on which the subjects were standing for 60 seconds with both feet and open eyes.

Results: When comparing the neutral and pes planus groups, none of the 17 time-distance and frequency based parameters showed any significant difference ( $\mathrm{p} \geq 0.169)$.

Conclusion: The results show that pes planus does not affect significantly standing balance; the differences (however not significant) between the two groups showed a poorer postural control in school-aged children with pes planus. It may be compensated by the increased ML dimension of the base of support.
\end{abstract}

Keywords: standing balance, center of pressure, children, pes planus, postural control

\section{Introduction}

Foot pressure basically determines the quality of our daily activities like working, walking and running, since the leg is responsible for the force which creates the motion and for supporting the human body during standing. Keeping the balance is a dynamic central nervous system controlled process, which could be affected by visual, vestibular and various orthopaedic lesions. ${ }^{1}$ According to the definition, standing balance (or static postural control) is the ability to keep the body „motionless” in given circumstances and in a given position, i.e., to stabilize and minimize the movements of the centre of mass (COM) ${ }^{2}$ With the help of the inverted pendulum principle it can be proved that during standing the movement of the centre of mass $(\mathrm{COM})$ can be characterized properly by the movement of the foot centre of pressure (COP). ${ }^{2}$ During standing, COP excursions are computed from the ground reaction forces, which provide an indication of postural control during quiet standing. From the two-dimensional COP coordinates obtained in the measurement interval numerous COP parameters can be calculated, which can be classified into time-domain and frequency-domain parameters. Despite the fact that the International Society for Posture and 
Gait Research has standardised many aspects of static stabilometry measurements in $2009^{3}$ a wide variety of parameters characterizing standing balance are still used by researchers also in the case of children. However, an individual with a high magnitude or velocity of COP excursions is thought to have impaired postural control.,

Legs, especially the fingers and the metatarsal area, play an important role in keeping the balance, pronated and supinated foot structures change the response time of the muscles around the talo-tibial joint., ${ }^{5,6}$ This suggests that any kind of change (height, flexibility) of the arches will also increase the possibility of a change in standing balance. The effect of foot structure change (pes rectus and pes planus) on standing balance is not a widely researched area. The foot structure change effect in the distribution of pressure on the surface of the sole. ${ }^{6}$ Pes planus (pronated flat-arched foot) may be associated with excessive subtalar joint pronation. Abnormal compensatory foot pronation may cause passive instability and hypermobility of the joints of the foot. ${ }^{5}$ Pes planus may therefore be unstable during weight bearing and might impair standing balance?

When Hertel et $\mathrm{al}^{8}$ compared young subjects with pes planus foot structures to those with pes rectus foot structures, they did not find any difference between the COP sway area nor between the average COP sway velocity values. At the same time, Cobb et $\mathrm{al}^{7}$ found significant differences in the pes planus group in case of anterior and posterior deformations during standing position measurements on one leg and with eyes closed (forefoot varus greater than $7^{\circ}$ ), so they assumed that this group had a poorer postural stability too. In their view, ${ }^{7}$ decreased stability associated with increased forefoot varus may be caused by decreased joint congruity and consequently an increased reliance on soft tissue structures for stability. Both studies ${ }^{7,8}$ classified the foot structure with a combination of weight bearing and nonweight bearing rearfoot and forefoot measures which caused a great variability in COP area and the average of the standard deviation of the ground reaction forces in both groups. This high variability could lead to opposite conclusions.

Cobb et $\mathrm{al}^{7}$ were confirmed by the research of Tsai et $\mathrm{al}^{9}$ and Cote et $\mathrm{al}^{10}$, because in these research works the values in anterior-posterior and in medial-lateral deformation and in COP sway and average COP sway velocity were significantly higher in the pes planus groups than in the neutral groups. According to $\mathrm{Al}$ Abdulwahab and Kahanatchu ${ }^{11}$ in case of young adults, pes planus does not affect standing balance since neither in the neutral nor in the pes planus groups were there any significant differences in COP sway velocity and foot pressure index. Sung et $\mathrm{al}^{12}$ found significant differences in kinetic stability index during single leg stance, if the eyes were closed. In our opinion, the very short measurement times (5-15 seconds) and the standing balance characterized by different parameters could be behind the controversy found in literature. This confirms too, that the sampling time could affect the size of the parameters. A recent study by Scoppa et $\mathrm{al}^{3}$ suggests short sampling time ( 25 seconds) for COP summary measures.

However, Carpenter et $\mathrm{al}^{13}$ recommended 60 seconds long interval time to ensure stable standard deviation of COP parameters for quiet standing trials when vision is available. No any research in literature was found to investigate the effect of pes planus on standing balance in elementary school-aged children. However, it would be very important because standing balance is constantly evolving, so it changes significantly in childhood. ${ }^{9}$ The objective of the present study is to determine 
the influence of pes planus on the standing balance of school-aged children during 60 -seconds-long bipedal standing with open eyes. The 60 seconds test ensures reliable use of frequency-based parameters. Nagymáté and Kiss ${ }^{14,15}$ studied many of time- distance and frequency based parameters with correlation analysis and variance analysis in different standing conditions to select the independent CoP parameters that sensitive enough to show differences between the different standing conditions. The authors of the present research recommended time-distance and frequency parameters as independent CoP parameter sets presented in Table 1. Timedistance type parameters characterise the COP movement by its excursions, spatial limits and speed of the motion. The frequency type parameters provide information of the frequency content of the COP motion, which is not trivial by the visual representation of the COP stabilogram, while the effect of the distance type parameters is rather visible. The recommendation of independent parameters is based on correlation and variance analysis of the COP parameters in different stance types and visual conditions. Since the parameters are independent, they can help us analyse the postural control of our subjects in different aspects. We hypothesized that, as in young adults, the standing balance parameters of the pes planus group differ significantly from the neutral group's parameters due to increased passive instability.

\section{Materials and Methods}

\section{Subjects}

From September 2015 to June 2016, 335 children (192 girls and 143 boys) were screened for the study in three state elementary schools in Region Szolnok (Hungary). The basic inclusion criterion for the subjects was 6-14 years of age. Conditions for exclusion included the following: any minor orthopaedic lesion of the lower limbs, surgery in the past 6 months, lower extremity injury, spine deformity (scoliosis, Scheuermann's disease), bad posture, cerebral palsy, cerebral concussion, visual or vestibular disorder, \pm 5 dioptres of vision correction, inner ear infection at the time of the examination, upper respiratory infection, or head cold. We also excluded those children who regularly performed exercises which improve balancing ability at high levels (ballet, sailing, tai chi).

In the orthopaedic examination during the first selection, by taking into account the inclusion and exclusion criteria, 12 children ( 3 girls and 9 boys) were excluded due to minor orthopaedic lesions, surgery, or injuries, 29 children (12 girls and 17 boys) due to scoliosis or Scheuermann's disease, 46 children (24 girls and 22 boys) due to bad posture, 3 boys due to cerebral concussions, visual or vestibular disorders, 2 boys due to \pm 5 dioptres visual correction and 6 children (2 girls and 4 boys) due to the regular performance of exercises which greatly improve balancing ability.

The research was authorized by the Research Ethics Committee of MÁV Hospital (license number: FI/5-93/2007). The parents of the subjects received detailed verbal and written information in each case before they signed the consent form. The current study conforms to the STROBE statement for reporting of casecontrol study. 16

\section{Classification of weight-bearing foot structures}

The remaining 237 children (151 girls, 86 boys) were divided into three groups according to their rearfoot-to-leg angles and the medial longitudinal arch angle which determine the foot structure. ${ }^{9}$ Tsai et $\mathrm{al}^{9}$ defined the rearfoot- 


\begin{tabular}{|c|c|c|}
\hline Parameter name & Dimension & Description \\
\hline \multicolumn{3}{|c|}{ Time-distance parameters } \\
\hline $\begin{array}{l}\text { Confidence ellipse } \\
\text { area (CE area) }\end{array}$ & $\mathrm{mm}^{2}$ & The area of the $95 \%$ confidence ellipse around the CoP trajectory \\
\hline $\begin{array}{l}\text { Confidence ellipse } \\
\text { axis ratio }(\mathrm{CE} \text { axis } \\
\text { ratio) }\end{array}$ & 1 & $\begin{array}{l}\text { The ratio between the major and the minor axes of the } 95 \% \text { confidence } \\
\text { ellipse that describes the shape of the CoP's trajectory expansion. }\end{array}$ \\
\hline Path length & $\mathrm{mm}$ & The length of the total CoP trajectory during the measurement. \\
\hline $\begin{array}{l}\text { Maximum path } \\
\text { velocity }\end{array}$ & $\mathrm{mm} / \mathrm{s}$ & $\begin{array}{l}\text { The filtered maximum distance between consecutive } \mathrm{CoP} \text { points } \\
\text { divided by the sampling interval. }\end{array}$ \\
\hline AP-ML range ratio & 1 & $\begin{array}{l}\text { The ratio of the largest CoP path expansions in the anteroposterior } \\
(\mathrm{AP}) \text { and mediolateral }(\mathrm{ML}) \text { directions that describes the relation of the } \\
\text { largest random errors of postural control between the two anatomical } \\
\text { directions. }\end{array}$ \\
\hline $\begin{array}{l}\text { Anterior }(\mathrm{AP}+) \\
\text { and Posterior (AP-) } \\
\text { maximum deviations }\end{array}$ & $\mathrm{mm}$ & $\begin{array}{l}\text { The maximum excursions in the anterior and posterior direction } \\
\text { relative to the average CoP point in the AP-ML plane }\end{array}$ \\
\hline $\begin{array}{l}\text { Largest amplitude } \\
\text { during balancing } \\
\text { (LA) }\end{array}$ & $\mathrm{mm}$ & $\begin{array}{l}\text { The largest continuous motion in both the } \mathrm{AP} \text { and the ML directions, } \\
\text { which are not necessarily equal to the corresponding CoP range. This } \\
\text { parameter is similar to the sub-movement size that was defined by }{ }^{22} \text { for } \\
\text { targeted CoP movements. }\end{array}$ \\
\hline \multicolumn{3}{|l|}{ Frequency parameters } \\
\hline $\begin{array}{l}\text { Frequency power } \\
\text { ratios between } \\
\text { low-medium and } \\
\text { medium-high } \\
\text { frequency bands } \\
\text { (LMR, MHR) }\end{array}$ & 1 & $\begin{array}{l}\text { Provide information about the power distribution of postural sway in } \\
\text { the frequency domain. The defined limits of the compared frequency } \\
\text { bands are low- }(0-0.3 \mathrm{~Hz}) \text { medium- }(0.3-1 \mathrm{~Hz}) \text { and high frequency } \\
(1-5 \mathrm{~Hz}) \text { bands. }\end{array}$ \\
\hline $\begin{array}{l}\text { Mean power } \\
\text { frequency }(\mathrm{MPF})\end{array}$ & $\mathrm{Hz}$ & $\begin{array}{l}\text { A weighted average frequency where } \mathrm{fj} \text { frequency components are } \\
\text { weighted by their Pj power. M is the number of frequency bins. MPF is } \\
\text { calculated as proposed by Oskoei and Hu } 24 \text {, according to the following } \\
\text { equation: } \boldsymbol{M P} \boldsymbol{F}=\sum_{\boldsymbol{j}=\mathbf{1}}^{\boldsymbol{M}} \boldsymbol{f}_{\boldsymbol{j}} \boldsymbol{P}_{\boldsymbol{j}} / \sum_{\boldsymbol{j}=\mathbf{1}}^{\boldsymbol{M}} \boldsymbol{P}_{\boldsymbol{j}}\end{array}$ \\
\hline $\begin{array}{l}\text { Spectral power ratio } \\
\text { (SPR) }\end{array}$ & 1 & $\begin{array}{l}\text { The ratio of the total spectral power in the AP direction and the total } \\
\text { spectral power in the ML direction. SPR characterizes the rate of power } \\
\text { distribution of postural sway frequencies in the AP/ML directions. }\end{array}$ \\
\hline \multicolumn{3}{|l|}{ Other } \\
\hline $\begin{array}{l}\text { Load distribution } \\
\text { difference (LDD) }\end{array}$ & $\%$ & $\begin{array}{l}\text { Shows the difference in the weight load on the lower limbs. This } \\
\text { parameter is not derived from CoP motion, but it is used by the original } \\
\text { Zebris WinPDMS software together with the CoP parameters and } \\
\text { proven to be very useful in biomechanical analyses. }{ }^{25}\end{array}$ \\
\hline
\end{tabular}

Table 1. Studied parameters 
to-leg angle as "the acute angle between the bisecting line of the calcaneus and the bisecting line of the distal one third of the leg”. They defined the medial longitudinal arch angle as "the obtuse angle between the line connecting the medial malleolus and navicular tuberosity, and the line connecting the navicular tuberosity and the most medial aspect of the first metatarsal head". The children in the neutral foot type group had rearfoot-to-leg angle between $3^{\circ}$ and $9^{\circ}$ and medial longitudinal arch angle between $134^{\circ}$ and $150^{\circ} .9$ In the pes planus (pronated) foot type group the children had rearfoot-to-leg angle greater than $9^{\circ}$ and medial longitudinal arch angles less than $134^{\circ} .9$ The third foot type group contained children with rearfoot-to-leg angle less than $3^{\circ}$ and medial longitudinal arch angles greater than $150^{\circ}$.

Standardization of the effect of a supinated foot structure was not in the scope of the study, therefore 28 children (20 girls, 8 boys) was excluded. Children with asymmetric foot structure (15 girls, 17 boys) were also excluded from the study. No children was found with rigidly pronated feet, which lesion was verified by the method of Tsai et al. ${ }^{9}$ In the end, two groups - one with 105 children (84 girls, 21 boys) with neutral foot type and another 72 children (32 girls, 40 boys) with pes planus (pronated, flat arched) foot type - were formed.

\section{Measurement method}

Standing balance measurements were carried out with a Zebris FDM-S multifunctional (320 mm x $470 \mathrm{~mm}$ measuring surface with 1504 pcs. load cells) (ZEBRIS GmbH, Isny, Germany) at the Biomechanical Laboratory of MÁV Hospital (Szolnok, Hungary). Vertical force distribution data was recorded by the Zebris WinPDMS processing software (v1.2) at $100 \mathrm{~Hz}$.
Each subject performed 60-second trials of barefoot bipedal stance with open eyes in light of day. During these measurements the subjects were in a bipedal natural standing position with a distance between the two ankle joint centres equal to the distance between the right and left anterior superior iliac spines. Both limbs were in full knee extension, heels were aligned in a line, feet were parallel and faced forward and arms were resting by the sides. The subjects focused on a black mark placed approximately $3 \mathrm{~m}$ away at eye level on a white wall in front of them. Correct feet placements had to be held throughout the examinations because changes thereof could affect stabilometry parameters. ${ }^{17}$ Every subject was asked to perform the required 60 -second bipedal standing as motionlessly as possible. Subjects were given 1 practice and 1 test trial, with 1 -minute rest periods between the consecutive trials. The trials were accepted only when the subjects maintained the required position for a minimum of 60 consecutive seconds. If they were not able not keep balance, they could repeat the measurements once more. If they could not succeed, they were excluded from the study. The 60 second long measuring ensures the calculation of frequency-type parameters ${ }^{13}$ and the difference could be easier detected during the 60 seconds-long bipedal standing with open eyes.

\section{Calculated parameters}

Further data processing and COP parameter calculations were carried out on exported raw measurement data in a custom application written in LabVIEW v2013 (National Instruments Inc., Austin, Texas). The calculated instantaneous COP coordinates were filtered with a Butterworth low-pass digital filter with a cut-off frequency of $10 \mathrm{~Hz}$ as recommended by Ruhe et al. ${ }^{18}$ From the COP position signals, a power spectrum was obtained using the Fast Fourier Transformation (FFT) with Hanning 
filtering window as recommended by Ruhe et al. ${ }^{18}$ Seventeen time-distance and frequency based parameters were calculated from COP position, which are recommended as indented parameters in, ${ }^{14,15}$ summarized in Table 1.

\section{Data analysis}

To analyse the impact of the pes planus, the average and standard deviation of the selected parameters were calculated for both groups as basic statistical features. Comparison of the two groups was carried out by a Student's t-test confirmed by F-tests to test the homogeneity of variance of the comparable parameters. The level of significance was set to 0.05 .

\section{Results}

The characteristics of the subjects in the two (neutral and pes planus) foot structure groups are shown in Table 2. Anthropometric data (age, height, weight) did not differ significantly, whereas the two parameters describing the foot structure (rearfoot-to-leg angle and medial longitudinal arch angle) differed significantly in the two groups.

All subjects (105) in the neutral group were able to perform the 60 -seconds-long bipedal open eyed standing at first time, whereas the test had to be repeated due to loss of balance in the case of 9 out of 75 children of the pes planus group, but nobody was excluded.

The statistics have been performed after excluding 8 outlier from the neutral group and 7 from the pes planus group. The average and standard values of the selected parameters in both groups are shown in Table 3. Many parameters show relatively large SD values compared to the mean value, however in most cases they do not differ between the two groups The F-test only shows significant difference in the standard deviations in the ML MHR parameter $(\mathrm{p}<0.01)$. According to the t-test no parameters show significant differences in the mean between the normal and the pes planus groups. This means that the postural control in the school aged children is not influenced by pes planus.

\section{Discussion}

The averages and standard deviations of the 17 independent parameters which characterize the balancing ability of 105 children ( 84 girls, 21 boys) with a neutral foot type and 72 children (32 girls, 40 boys) with pes planus (pronated, flat arched) are shown in Table 3. According to our knowledge, this is the first article about standing balance analysed with distance, time and frequency based parameters calculated from the results of 60-seconds-long

\begin{tabular}{|c|c|c|c|}
\hline & & $\begin{array}{c}\text { neutral group } \\
\mathrm{N}=105 \\
\text { (84 girls, } 21 \text { boys) }\end{array}$ & $\begin{array}{c}\text { pes planus (pronated) group } \\
N=72 \\
\text { (32 girls, } 40 \text { boys) }\end{array}$ \\
\hline \multicolumn{2}{|l|}{ age (years) } & range: $6-14$ & range: $6-14$ \\
\hline \multicolumn{2}{|l|}{ weight (kg) } & $48.5 \pm 14.88$ & $48.3 \pm 14.59$ \\
\hline \multicolumn{2}{|l|}{ height (m) } & $1.53 \pm 0.13$ & $1.54 \pm 0.10$ \\
\hline \multirow{2}{*}{ rearfoot-to-leg angle (deg) } & left & $5.5 \pm 1.6$ & $12.3 \pm 1.6$ \\
\hline & right & $5.6 \pm 1.8$ & $11.9 \pm 1.7$ \\
\hline \multirow{2}{*}{$\begin{array}{c}\text { medial longitudinal arch } \\
\text { angle (deg) }\end{array}$} & left & $146.1 \pm 1.9$ & $129.1 \pm 2.1$ \\
\hline & right & $145.6 \pm 2.1$ & $129.9 \pm 2.2$ \\
\hline
\end{tabular}

Table 2. Data of the subjects. Values are mean \pm SD unless otherwise indicated 
measurements at children with neutral and with pes planus foot type. It means, there were no reference values for many examined timedistance and frequency-based parameters, because those parameters were calculated from only 30 -seconds-long measurements.

Table 3 shows that many averaged parameters have relatively large SD values. This is due to the intra-subject variability of these COP measures. ${ }^{19}$ Papers dealing with the reliability of these parameters reported similarly large relative SD values together with high reliability of the parameters described by Intraclass Correlation Coefficient (ICC).${ }^{19}$ For instance, Laroche et al ${ }^{19}$ among many similarly behaving parameter reported $543 \mathrm{~mm}$ (95\% Confidence interval: $186-900 \mathrm{~mm}$ ) for COP path length with high reliability of ICC $=0.85$. The inter- subject variability of both groups are similar (see F-test values in Table 2). In our opinion the higher deviation values compared to the mean values could be caused by the differences in the children's neurological maturity. ${ }^{20}$ The parameters of balancing ability vary with age, ${ }^{20}$ so the wide range of age (6 to 14 years) of the subjects can cause high SD values. In the neutral group (Table 2) the maximum velocity, the $95 \%$ CE Area ${ }^{20}$ and the path length ${ }^{21}$ are in good agreement with the results in young subjects presented in literature.

The aim of the present study was to investigate whether pes planus changes standing balance. By studying the literature, it can be stated that standing balance measurements in children with pes planus foot structure have not been performed yet. We hypothesized that the

\begin{tabular}{|l|r|r|r|r|r|}
\hline & \multicolumn{1}{|c|}{$\begin{array}{c}\text { neutral group } \\
\mathrm{N}=105\end{array}$} & $\begin{array}{c}\text { pes planus } \\
\text { (pronated) group } \\
\mathrm{N}=72\end{array}$ & $\begin{array}{c}\text { mean } \\
\text { difference }\end{array}$ & $\begin{array}{c}\text { F-test } \\
\mathrm{p}\end{array}$ & $\begin{array}{c}\text { t-test } \\
\mathrm{p}\end{array}$ \\
\hline 95\% CE axis ratio & $1.7 \pm 0.571$ & $1.691 \pm 0.476$ & 0.010 & 0.104 & 0.905 \\
\hline 95\% CE area $(\mathrm{mm})$ & $262.466 \pm 183.086$ & $244.705 \pm 186.087$ & 17.761 & 0.871 & 0.530 \\
\hline Path length $(\mathrm{mm})$ & $855.542 \pm 254.855$ & $854.788 \pm 222.625$ & 0.754 & 0.225 & 0.984 \\
\hline Max velocity $(\mathrm{mm} / \mathrm{s})$ & $115.359 \pm 50.955$ & $117.106 \pm 56.328$ & 1.747 & 0.349 & 0.830 \\
\hline AP-ML range ratio & $1.262 \pm 0.486$ & $1.322 \pm 0.501$ & 0.060 & 0.765 & 0.427 \\
\hline LDD $(\%)$ & $6.202 \pm 5.146$ & $7.19 \pm 5.912$ & 0.988 & 0.196 & 0.239 \\
\hline AP LA $(\mathrm{mm})$ & $28.522 \pm 13.076$ & $25.952 \pm 10.656$ & 2.570 & 0.067 & 0.169 \\
\hline ML LA $(\mathrm{mm})$ & $24.577 \pm 10.888$ & $23.692 \pm 12.023$ & 0.885 & 0.354 & 0.611 \\
\hline A max.dev $(\mathrm{mm})$ & $26.59 \pm 11.072$ & $25.068 \pm 11.071$ & 1.522 & 0.990 & 0.370 \\
\hline P max.dev $(\mathrm{mm})$ & $26.648 \pm 11.36$ & $26.723 \pm 12.438$ & 0.074 & 0.396 & 0.967 \\
\hline AP MPF $(\mathrm{Hz})$ & $0.147 \pm 0.058$ & $0.155 \pm 0.063$ & 0.008 & 0.446 & 0.404 \\
\hline ML MPF $(\mathrm{Hz})$ & $0.192 \pm 0.074$ & $0.191 \pm 0.071$ & 0.001 & 0.661 & 0.933 \\
\hline SPR & $2.005 \pm 1.521$ & $2.001 \pm 1.762$ & 0.005 & 0.171 & 0.985 \\
\hline AP LMR & $11.333 \pm 9.557$ & $9.868 \pm 8.605$ & 1.464 & 0.347 & 0.299 \\
\hline AP MHR & $11.978 \pm 6.308$ & $11.994 \pm 7.386$ & 0.017 & 0.142 & 0.987 \\
\hline ML LMR & $6.79 \pm 5.962$ & $5.912 \pm 5.144$ & 0.878 & 0.186 & 0.311 \\
\hline ML MHR & $11.455 \pm 4.85$ & $13.297 \pm 8.329$ & 1.842 & $<0.010$ & 0.094 \\
\hline
\end{tabular}

Table 3. Statistical comparison of the neutral and pes planus groups' standing balance based on COP parameters. Values are mean \pm SD unless otherwise indicated.

CE: confidence ellipse, AP: anteroposterior, ML: mediolateral, LDD: load distribution difference between legs, LA: largest amplitude, A: anterior, P: posterior, max dev: maximum deviation, MPF: mean power frequency, SPR: spectral power ratio, LMR: low-medium band power ratio MHR: medium-high frequency band power ratio 
children with pes planus foot structures would have had significant poorer standing balance than children with neutral feet, because of reduced stability in the foot joints. ${ }^{7}$ Based on the results of 17 independent parameters, the standing balance of children with pes planus is poorer compared to children with neutral feet, however no significant difference was found between the two groups at any parameter ( $\mathrm{p} \geq 0.169$ (Table 2). It means, our hypothesis could not be justified.

The differences (however not significant) between the two groups (Table 3) showed that school-aged children with pes planus could have a poorer postural control, which may be compensated by the increased ML dimension of the base of support coincided with foot pronation. The advantage of increased ML dimension of the base of support is strengthened by the decreased largest amplitude in direction ML (ML LA) and by the decreased mean power frequency in ML direction (ML MPF) in subjects with pes planus compared to neutral group (Table 3). It can be assumed that children's flexible foot structure and increased ML dimension of the base of support are suitable for correction, during the longer measurement time (60 seconds). It means that the different balancing mechanism of young adults has not developed in children yet. The open eyes and bipedal stance could contribute to the compensatory mechanism too. They could be the reason why no significant differences were found in any of the 17 independent parameters.

Controversial results were found comparing standing balance in young adults with pes planus and neutral foot structures. Hertel et $\mathrm{al}^{8}$ did not find significant differences even in the case of single leg measurements in COP area and COP speed parameters, which was confirmed by the research of Abdulwahab and Kahanatchu. ${ }^{11}$ The results are strengthened by the research of Sung et $a{ }^{12}$ because they did not find significant differences in kinetic stability index during single leg stance with open eyes. Cobb et al, ${ }^{7}$ Cote et $\mathrm{al}^{10}$ and Tsai et $\mathrm{al}^{9}$ found contradictory results, but the features of the standing balance were produced with very short, 5-10-15 second long, one-legged, open and closed eyed measurements.

The limit of study was the fact that the examinations were not performed during single leg stance with open and closed eyes as well as during bipedal stance with closed eyes due to accident prevention considerations.

The present study is unique because the values of the parameters characterizing standing balance were determined according to various criteria in a large number of children with pes planus (79 children) and of neutral foot structures (105 children). Based on the statistical analysis of the results (Table 3), pes planus does not affect significantly standing balance because none of the 17 parameters show any significant difference $(\mathrm{p} \geq 0.169)$.

The differences (however not significant) between the two groups (Table 3) showed that school-aged children with pes planus could have a poorer postural control. Structural changes in the foot did not yet significantly appear in the parameters which describe the balancing ability, however the results of these studies could be highlighted that the pronated foot in elementary school-aged children may be appropriate special clinical improve (special shoe wear, physiotherapy) to improve postural control. 
The authors would like to express their gratitude to Ildikó Nagy and to Gábor Szabó physiotherapists for their valuable work in the biomechanical measurements. This work was supported by the Hungarian Scientific Research Fund OTKA [grant number K115894]. The research reported in this paper was supported by the Higher Education Excellence Program of the Ministry of Human Capacities in the frame of Biotechnology research area of Budapest University of Technology and Economics (BME FIKP-BIO)

\section{REFERENCES}

1. Winter DA, Patla AE, Prince F, Ishac M, Gielo-Perczak K. Stiffness control of balance in quiet standing. J Neurophysiol. 1998;80(3):1211-21.

2. Hasan SS, Robin DW, Szurkus DC, Ashmead DH, Peterson SW, Shiavi RG. Simultaneous measurement of body center of pressure and center of gravity during upright stance. Part II: Amplitude and frequency data. Gait Posture. 1996 Jan 31;4(1):11-20.

3. Scoppa F, Capra R, Gallamini M, Shiffer R. Clinical stabilometry standardization. Basic definitions - Acquisition interval - Sampling frequency. Gait Posture. 2013;37(2):290-2.

4. Verbecque E, Vereeck L, Hallemans A. Postural sway in children: A literature review. Gait Posture. 2016 Sep;49:402-10.

5. Franco AH. Pes Cavus and Pes Planus. Phys Ther. 1987 May 1;67(5):688-94.

6. PaukJ, Daunoraviciene K, Ihnatouski M, Griskevicius J, Raso J V. Analysis of the plantar pressure distribution in children with foot deformities. Acta Bioeng Biomech. 2010;12(1):29-34.

7. Cobb SC, Tis LL, Johnson BF, Higbie EJ. The effect of forefoot varus on postural stability. J Orthop Sports Phys Ther. 2004;34:79-85.

8. Hertel J, Gay MR, Denegar CR. Differences in postural control during single-leg stance among healthy individuals with different foot types. J Athl Train. 2002;37(2):129-32.

9. Tsai L-C, Yu B, Mercer VS, Gross MT. Comparison of different structural foot types for measures of standing postural control. J Orthop Sports Phys Ther. 2006;36(12):942-53.

10. Cote KP, Brunet ME, Gansneder BM, Shultz SJ. Effects of pronated and supinated foot postures on static and dynamic postural stability. J Athl Train. 2005;40(1):41-6.

11. Al Abdulwahab SS, Kachanathu SJ. The effect of various degrees of foot posture on standing balance in a healthy adult population. Somatosens Mot Res. 2015 Jul 3;32(3):172-6.

12. Sung PS, Zipple JT, Andraka JM, Danial P. The kinetic and kinematic stability measures in healthy adult subjects with and without flat foot. Foot. 2017;30:21-6.

13. Carpenter MG, Frank JS, Winter DA, Peysar GW. Sampling duration effects on centre of pressure summary measures. Gait Posture. 2001 Feb;13(1):35-40.

14. Nagymáté G, Kiss RM. Parameter reduction in the frequency analysis of center of pressure in stabilometry. Period Polytech Mech Eng. 2016;60(4):238-46.

15. Nagymáté G, Kiss RM. Replacing Redundant Stabilometry Parameters with Ratio and Maximum Deviation Parameters. In: Proceedings of the 12th IASTED International Conference on Biomedical Engineering. Calgary,AB,Canada: ACTAPRESS; 2016. p. 140-4.

16. von Elm E, Altman DG, Egger M, Pocock SJ, Gøtzsche PC, Vandenbroucke JP. The Strengthening the Reporting of Observational Studies in Epidemiology (STROBE) Statement: Guidelines for reporting observational studies. Prev Med (Baltim). 2007 Oct;45(4):247-51.

17. Chiari L, Rocchi L, Cappello A. Stabilometric parameters are affected by anthropometry and foot placement. Clin Biomech. 2002 Nov;17(910):666-77.

18. Ruhe A, Fejer RR, Walker B. The test-retest reli- 
ability of centre of pressure measures in bipedal static task conditions - A systematic review of the literature. Gait Posture. 2010;32(4):436-45.

19. Laroche D, Kubicki A, Stapley PJ, Gremeaux V, Mazalovic K, Maillefert JF, et al. Test-retest reliability and responsiveness of centre of pressure measurements in patients with hip osteoarthritis. Osteoarthr Cartil. 2015;23(8):1357-66.

20. Hsu YS, Kuan CC, Young YH. Assessing the development of balance function in children using stabilometry. Int J Pediatr Otorhinolaryngol. 2009;73(5):737-40.

21. Sakaguchi M, Taguchi K, Miyashita Y, Katsuno S. Changes with aging in head and center of foot pressure sway in children. Int J Pediatr Otorhinolaryngol. 1994 Apr;29(2):101-9.

22. Hernandez ME, Ashton-Miller JA, Alexander
NB. Age-related changes in speed and accuracy during rapid targeted center of pressure movements near the posterior limit of the base of support. Clin Biomech. 2012;27(9):910-6.

23. Nagy E, Toth K, Janositz G, Kovacs G, Feher-Kiss A, Angyan L, et al. Postural control in athletes participating in an ironman triathlon. Eur J Appl Physiol. 2004;92(4-5):407-13.

24. Oskoei $M A, H u H$. Support vector machine-based classification scheme for myoelectric control applied to upper limb. IEEE Trans Biomed Eng. 2008 Aug;55(8):1956-65.

25. Duffell LD, Gulati V, Southgate DFL, McGregor AH. Measuring body weight distribution during sit-to-stand in patients with early knee osteoarthritis. Gait Posture. 2013 Sep;38(4):745-50.

\section{Mária Takács}

Department of Orthopedics, MÁV Hospital Szolnok H-5000, Szolnok, Verseghy F. str. 6-8, Tel.: (+36) 56524633 